\title{
Feature detection and tracking in optical flow on non-flat manifolds
}

ARTICLE in PATTERN RECOGNITION LETTERS • NOVEMBER 2011

Impact Factor: 1.55 · DOI: 10.1016/j.patrec.2011.09.017 · Source: DBLP

CITATIONS

2

4 AUTHORS, INCLUDING:

Sheraz Khan

Harvard Medical School

18 PUBLICATIONS 97 CITATIONS

SEE PROFILE

Sylvain Baillet

McGill University

156 PUBLICATIONS 4,176 CITATIONS

SEE PROFILE
Julien Lefèvre

Aix-Marseille Université

37 PUBLICATIONS 208 CITATIONS

SEE PROFILE 


\title{
Feature Detection and Tracking in Optical Flow on Non-Flat Manifolds
}

\author{
Sheraz Khan ${ }^{\mathrm{a}, \mathrm{c}, \mathrm{d}, *}$, Julien Lefevre $^{\mathrm{b}}$, Habib Ammari ${ }^{\mathrm{c}}$, Sylvain Baillet ${ }^{\mathrm{a}}$ \\ ${ }^{a}$ Department of Neurology, Medical College of Wisconsin, Milwaukee, USA. \\ ${ }^{b}$ University of the Mediterranean Aix-Marseille II / LSIS CNRS 6168, Marseille, France. \\ ${ }^{c}$ Center of Applied Mathematics, Ecole Polytechnique France. \\ ${ }^{d}$ Department of Neurology, MGH/ Harvard Medical School, Boston, USA.
}

Keywords: Optical Flow, Helmholtz-Hodge Decomposition, Feature Detection, Image Processing, Riemannian Formalism, Vector Field.

\begin{abstract}
Optical flow is a classical tool to estimate velocity vector fields from objects on a manifold. The Helmholtz-Hodge Decomposition (HHD) of vector fields has been used to isolate rotational and divergential features in vector fields, such as vortices in vector fields. However, the existing HHD techniques operate on flat, 2D domains, which is non-adequate to many potential applications. In this paper, we extend the Helmholtz-Hodge decomposition to vector fields defined over any arbitrary surface manifolds. This is achieved using a Riemannian variational formalism. We illustrate the proposed methodology with the decomposition of optical flow vector fields defined on a variety of surface objects using synthetic and experimental data.
\end{abstract}

\footnotetext{
*Mailing Address: Department of Neurology, Massachusetts General Hospital, Harvard Medical School, Boston, MA 02129, Phone: 617-643-5634, Fax 617-948-5966
} 


\section{Introduction}

Optical flow is the apparent motion due to temporal variations in the observed pattern of brightness. Under certain conditions, optical flow is an adequate match of the motion field of the displacement of an object, Horn and Schunck (1981). In most applications, the optical flow is estimated on 2D, i.e. flat, surface manifolds. This is problematic when the object of interest is moving on a non-flat domain as in the case of flow turbulence over general surfaces. Recently, we introduced a new variational framework to estimate optical-flow vector fields on non-flat surfaces using a Riemannian formulation, Lefèvre and Baillet (2008). Here we broaden this framework to detect patterns - such as sources, sinks and traveling waves - in the spatial distribution of optical flow vector fields by extending the definition of the Helmholtz-Hodge Decomposition (HHD) to non-flat domains. This contribution uses the main framework of vector fields on differential geomtery and its implementation by Finite Element Method (FEM) from Lefèvre and Baillet (2008). To make this article self contained we revisit important concepts in Section II.

The Helmholtz-Hodge Decomposition, Chorin and Marsden (1993), is a technique to decompose a $2 \mathrm{D}$ or $3 \mathrm{D}$ continuous vector field into the sum of the three following components:

- a non-rotational part, deriving from the gradient of a scalar potential $U$;

- a non-diverging part, deriving from the rotational of a scalar or vector potential $A$ in $2 \mathrm{D}$ or $3 \mathrm{D}$, respectively;

- a harmonic part $\mathbf{H}$, whose Laplacian vanishes. 
From these components, it has been suggested that features of interest, singularities or vanishing points of the vector field could be detected. These singularities are related to features of a more direct, physical nature, Guo et al. (2005) such as sources, sinks and vortices (see Section 3.3). Although feature analysis of vector fields have multiple practical applications, only a few studies have specifically addressed their detection and visualization so far, Scheuermann and Tricoche (2005).

Furthermore, in most of the current literature, HHD has been described on flat, 2D domains, Guo et al. (2005) or in full 3D geometry, Tong et al. (2003). However, multiple fields of application - such as: experimental fluid dynamics and turbulence, Corpetti et al. (2003), Palit et al. (2005); physiological modeling, Guo et al. (2006); structural and functional neuroimaging, Lefevre et al. (2009), Khan et al. (2009) and the compressed representation of large, distributed vector fields, Scheuermann and Tricoche (2005) - would benefit from a generic and principled approach to the HHD of vector fields on surface manifolds. Some authors have suggested that approximations of surface-based HHD could be achieved on polyhedral surfaces, Polthier and Preuss (2003), using the local Euclidean geometry of the manifold, which is problematic with highly curved surface supports.

In the context of optical flow, we have shown in Lefèvre and Baillet (2008) that the surface curvature needs to be explicitly considered for the robust estimation of vector fields within the tangent bundle of the surface support. Moreover, we have shown that results on convergence of the numerical estimation of the vector field can be modified by the non-flatness properties of the surface. Therefore HHD frameworks defines on the euclidean domain, Polthier and Preuss (2003), will not be adequate on curved manifolds. We have worked on mesh representation of 
surfaces but we have to mention very recent and promising methods, that are still applied to euclidian domains at the moment but which are meshless, Petronetto et al. (2009).

In this article, we extend these results by redefining the HHD of vector fields in the context of Riemannian geometry for non flat manifolds to avoid shortcoming of previous methods, Polthier and Preuss (2003), Petronetto et al. (2009) defined on euclidean geometry.

The aim of this contribution is therefore twofold: first, we redefine the HHD on 2-Riemannian manifolds and second, we highlight its application to the detection of features in vector fields of the optical flow defined on general surfaces. Section II briefly revisits the concept of differential geometry and estimation of optical flow on non-flat surface domains. Section III introduces the new framework for HHD on 2-Riemannian manifolds. A variety of results are presented in section IV.

The methods discussed here were implemented in Matlab and are available for download as a plugin of the Brainstorm academic software for electromagnetic brain imaging (http://neuroimage.usc.edu/brainstorm). The code is implemented in a multi-threaded way, to take advantage of multicore processors available in current systems.

\section{Mathematical backgrounds}

This subsection is for introductory purposes; a more detailed description of its contents is in Do Carmo (1993) and Lefèvre and Baillet (2008). 


\subsection{Differential geometry}

In the following section we will consider $\mathcal{M}$ a surface (or 2-Riemannian manifold) equipped by a metric or scalar product $g(.,$.$) that allows to measure distances$ and angles on the surface.

$\mathcal{M}$ can be parameterized by local charts $\left(x_{1}, x_{2}\right)$. Thus, it is possible to obtain a normal vector at each point:

$$
\mathbf{n}_{p}=\frac{\partial}{\partial x_{1}} \times \frac{\partial}{\partial x_{2}}
$$

Note that $\mathbf{n}_{p}$ does not depend on the choice of the parametrization $\left(x_{1}, x_{2}\right)$.

Given $U: \mathcal{M} \rightarrow \mathbb{R}$ a function defined on the surface, we can obtain the differential $d U: T \mathcal{M} \rightarrow \mathbb{R}$ which acts on $T \mathcal{M}$ the space of vector fields. We have:

$$
d U\left(v_{1}, v_{2}\right)=\frac{\partial U}{\partial x_{1}} v_{1}+\frac{\partial U}{\partial x_{2}} v_{2}
$$

Then we define the gradient and divergence operators through duality:

$$
\mathrm{d} U(\mathbf{V})=g\left(\nabla_{\mathscr{M}} U, \mathbf{V}\right), \quad \int_{\mathscr{M}} U \operatorname{div}_{\mathcal{M}} \mathbf{H}=-\int_{\mathscr{M}} g\left(\mathbf{H}, \nabla_{\mathscr{M}} U\right)
$$

It is important to note that gradient and divergence are independent of the parametrization.

Lastly, we have to define two functional spaces that will be useful for a theorem in part 3. We define $H^{1}(\mathcal{M})$ as the space of differentiable functions on the manifold $\mathscr{M}$ and $\Gamma^{1}(\mathcal{M})$ the space of vector field which satisfy good properties of regularity (see Druet et al. (2004), Lefèvre and Baillet (2008) for more details). 


\subsection{Optical flow}

Under the seminal hypothesis of the conservation of a scalar field $I$ along streamlines the optical flow $\mathbf{V}$ is a vector field which satisfies:

$$
\partial_{t} I+g\left(\mathbf{V}, \nabla_{\mathcal{M}} I\right)=0
$$

Note that the scalar product $g(\cdot, \cdot)$ is modified by the local curvature of $\mathcal{M}$, the domain of interest. The solution to Eq. 1 is not unique as long as the components of $\mathbf{V}(p, t)$ orthogonal to $\nabla_{\mathcal{M}} I$ are left unconstrained. This so-called 'aperture problem' has been addressed by a large number of methods using e.g., regularization approaches. These latter may be formalized as the minimization problem of an energy functional, which both includes the regularity of the solution and the agreement to the model:

$$
\mathcal{E}(\mathbf{V})=\int_{\mathcal{M}}\left[\frac{\partial I}{\partial t}+g\left(\mathbf{V}, \nabla_{\mathcal{M}} I\right)\right]^{2} \mathrm{~d} \mu+\lambda \int_{\mathscr{M}} C(\mathbf{V}) \mathrm{d} \mu
$$

where $d \mu$ is a volume form of the manifold $\mathcal{M}$.

Here we have considered the following regularity factor, which operates quadratically on the generalized gradient of the expected vector field:

$$
\mathcal{C}(\mathbf{V})=\operatorname{Tr}\left({ }^{t} \nabla \mathbf{V} \cdot \nabla \mathbf{V}\right)
$$

Note that in order to be an intrinsic tensor, the gradient of a vector field must be defined as the covariant derivative associated to the manifold $\mathcal{M}$. We refer to the concepts of differential geometry for more information on this notion Do Carmo (1993). 


\section{Helmholtz-Hodge decomposition on 2-Riemannian manifold}

We now introduce an extended framework to perform HHD on surfaces and show that it can be applied to any vector field defined on a 2-Riemannian manifold, $\mathcal{M}$.

\subsection{Definitions and theorem}

First we define scalar and vectorial curls by:

$$
\begin{array}{r}
\operatorname{Curl}_{\mathscr{M}}: H^{1}(\mathscr{M}) \rightarrow \Gamma^{1}(\mathcal{M}) \quad \operatorname{curl}_{\mathscr{M}}: \Gamma^{1}(\mathscr{M}) \rightarrow H^{1}(\mathcal{M}) \\
\mathbf{C u r l}_{\mathcal{M}} A=\nabla_{\mathscr{M}} A \times \mathbf{n}, \quad \operatorname{curl}_{\mathcal{M}} \mathbf{H}=\operatorname{div}_{\mathcal{M}}(\mathbf{H} \times \mathbf{n}) .
\end{array}
$$

These formulas provide intrinsic expressions that do not depend on the parametrization of the surface.

We reformulate the results established in Polthier and Preuss (2003). We assume in the following that $\mathcal{M}$ is a closed manifold, i.e. it has no boundaries. Given $\mathbf{V}$ a vector field in $\Gamma^{1}(\mathcal{M})$, there exists functions $U$ and $A$ (up to an additive constant) in $H^{1}(\mathcal{M})$ and a vector field $\mathbf{H}$ in $\Gamma^{1}(\mathcal{M})$ such that:

$$
\mathbf{V}=\nabla_{\mathcal{M}} U+\mathbf{C u r l}_{\mathcal{M}} A+\mathbf{H}
$$

where

$$
\begin{gathered}
\operatorname{curl}_{\mathscr{M}}\left(\nabla_{\mathcal{M}} U\right)=0, \quad \operatorname{div}_{\mathcal{M}}\left(\operatorname{Curl}_{\mathscr{M}} A\right)=0, \\
\operatorname{div}_{\mathcal{M}} \mathbf{H}=0, \quad \operatorname{curl}_{\mathcal{M}} \mathbf{H}=0 .
\end{gathered}
$$


To prove this theorem we show how $U$ and $A$ may be constructed.

Following classical constructions, we consider $U$ and $A$ as minimizers of the two following convex functionals (there are not necessary unique):

$$
\begin{gathered}
\int_{\mathcal{M}}\left\|\mathbf{V}-\nabla_{\mathcal{M}} U\right\|^{2} \mathrm{~d} \mu, \\
\int_{\mathcal{M}}\left\|\mathbf{V}-\mathbf{C u r l}_{\mathcal{M}} A\right\|^{2} \mathrm{~d} \mu,
\end{gathered}
$$

where $\|$.$\| is the norm associated to the Riemannian metric g(\cdot, \cdot)$.

These two functionals carry a global minimum on $H^{1}(\mathcal{M})$, which satisfies:

$$
\begin{aligned}
\forall \phi \in H^{1}(\mathcal{M}), \int_{\mathscr{M}} g\left(\mathbf{V}, \nabla_{\mathcal{M}} \phi\right) \mathrm{d} \mu & =\int_{\mathcal{M}} g\left(\nabla_{\mathcal{M}} U, \nabla_{\mathcal{M}} \phi\right) \mathrm{d} \mu, \\
\forall \phi \in H^{1}(\mathcal{M}), \int_{\mathscr{M}} g\left(\mathbf{V}, \mathbf{C u r l}_{\mathscr{M}} \phi\right) \mathrm{d} \mu & =\int_{\mathcal{M}} g\left(\mathbf{C u r l}_{\mathcal{M}} A, \mathbf{C u r l}_{\mathcal{M}} \phi\right) \mathrm{d} \mu .
\end{aligned}
$$

These two equations will be very important for numerical computations. The end of the proof can be found in the Appendix.

\subsection{Discretization}

The two equations, (7) and (8) are crucial since they provide the path to numerical implementations when $H^{1}(\mathcal{M})$ is approximated by a subspace of finite elements (e.g., continuous linear piecewise functions).

We now detail the numerical implementation of Eqs. (9) and (10), which are defined on a surface tessellation $\hat{\mathcal{M}}$ that approximates the ideal manifold. Let $N$ be the number of nodes in the tessellation, respectively (Fig. 1).

Following the Finite Element Method (FEM), we define $N$ continuous piecewise affine functions $\phi_{i}$, whose values are 1 at node $i$ and 0 at all other triangle nodes. 

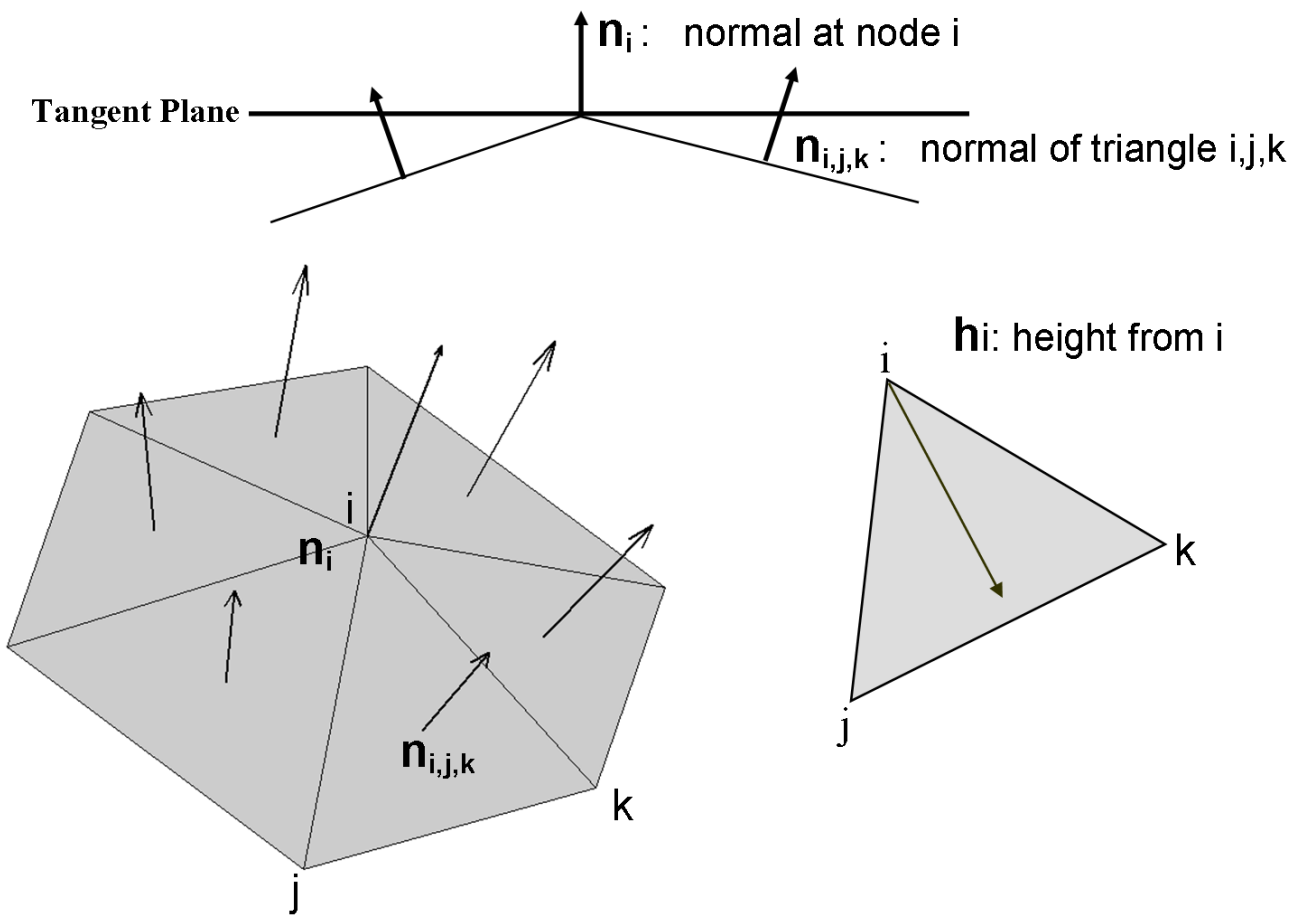

Figure 1: Illustration of local computations and associated definitions from FEM on a triangular surface mesh.

Indeed, with this given basis functions $\left(\phi_{1}, \ldots, \phi_{n}\right)$, we can write $\mathbf{U}=\left(U_{1}, \ldots, U_{n}\right)^{T}$, $\mathbf{A}=\left(A_{1}, \ldots, A_{n}\right)^{T}$, and Eqs; (7) and (8) read in a matrix way:

$$
\begin{aligned}
{\left[\int_{\mathcal{M}} g\left(\nabla_{\mathcal{M}} \phi_{i}, \nabla_{\mathscr{M}} \phi_{j}\right)\right]_{i, j} \mathbf{U} } & =\left[\int_{\mathscr{M}} g\left(\mathbf{V}, \nabla_{\mathscr{M}} \phi_{i}\right)\right]_{i} \\
{\left[\int_{\mathscr{M}} g\left(\mathbf{C u r l}_{\mathscr{M}} \phi_{i}, \operatorname{Curl}_{\mathscr{M}} \phi_{j}\right)\right]_{i, j} \mathbf{A} } & =\left[\int_{\mathscr{M}} g\left(\mathbf{V}, \operatorname{Curl}_{\mathscr{M}} \phi_{i}\right)\right]_{i} .
\end{aligned}
$$

where $[.]_{i, j}$ and $[.]_{i}$ represent a square matrix of size $N$ and a column vector of size $N$. 
The harmonic component $\mathbf{H}$ of the vector field $\mathbf{V}$ is obtained as:

$$
\mathbf{H}=\mathbf{V}-\nabla_{\mathcal{M}} U-\mathbf{C u r l}_{\mathscr{M}} A .
$$

Gradient of each basis function is constant on each triangle and can be simply computed with geometrical quantities (see Lefèvre and Baillet (2008)). So, Eq. (9) reads:

$$
\left[\sum_{T \ni i, j} \frac{\mathbf{h}_{\mathbf{i}}}{\left\|\mathbf{h}_{\mathbf{i}}\right\|^{2}} \cdot \frac{\mathbf{h}_{\mathbf{j}}}{\left\|\mathbf{h}_{\mathbf{j}}\right\|^{2}} \mathcal{A}(T)\right]_{i, j} \mathbf{U}=\left[\sum_{T \ni i} \mathcal{A}(T) \mathbf{V} \cdot \frac{\mathbf{h}_{\mathbf{i}}}{\left\|\mathbf{h}_{\mathbf{i}}\right\|^{2}}\right]_{i}
$$

where $\mathbf{h}_{\mathbf{i}}$ is the height taken from $i$ in the triangle $T, \mathcal{A}(T)$ is the surface area of triangle $T$.

Similarly, Eq. (10) is discretized as follows:

$$
\left[\sum_{T \ni i, j}\left(\frac{\mathbf{h}_{\mathbf{i}}}{\left\|\mathbf{h}_{\mathbf{i}}\right\|^{2}} \times \mathbf{n}\right) \cdot\left(\frac{\mathbf{h}_{\mathbf{j}}}{\left\|\mathbf{h}_{\mathbf{j}}\right\|^{2}} \times \mathbf{n}\right) \mathcal{A}(T)\right]_{i, j} \mathbf{A}=\left[\sum_{T \ni i} \mathcal{A}(T) \mathbf{V} \cdot\left(\frac{\mathbf{h}_{\mathbf{i}}}{\left\|\mathbf{h}_{\mathbf{i}}\right\|^{2}} \times \mathbf{n}\right)\right]_{i}
$$

where $\mathbf{n}$ is the normal to triangle $T$.

\subsection{Feature detection as critical points of potentials}

The critical points of a vector field are often classified depending on the eigenvalues of the Jacobian matrix at a point in a vector field. In the present case however, critical points of the flow may be defined as local extrema of the divergencefree potential $A$ (representing rotation) and curl-free potential $U$ (representing divergence). This definition is coherent with previous works such as Tong et al. (2003). Feature detection, as critical points in global potential fields is much less sensitive to noise in the data Tong et al. (2003) and therefore is less prone to false 
positives, when compared to methods based on the extraction of local Jacobian eigenvalues, Mann and Rockwood (2002).

A sink (respectively, a source) is defined as a local maximum (respectively, minimum) of $U$. Counterclockwise and clockwise vortex objects are featured by local maximum and minimum of $A$, respectively. Since $\mathbf{H}$ a vector field which has zero divergence and zero curl, traveling objects may be detected through vector elements bearing the highest norms in the $\mathbf{H}$ vector field. 


\section{Results}

The new methodology is first evaluated by detecting sources and sinks on the surface of a simplest curved manifold i.e. sphere, In Fig. 4 HHD is applied on spherical manifold having a rotating vector field (green arrows). Possible time domain of this vector field can be considered as hurricane (vortex) on the surface of the earth as viewed from weather satellites.

HHD decomposition is then applied on this vector field. Vortices in rotating vector fields are identified by critical points in $A$ and shown in a colormap overlapping with the vector field. The counter-clockwise rotating vector fields, vortex is identified by maxima in $A$, whereas the clockwise rotating vector fields, vortex is identified by minima in $A$.

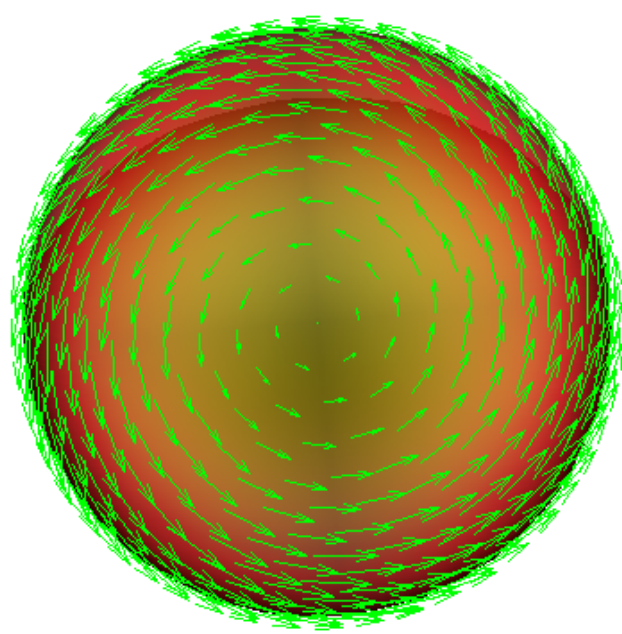

(a)

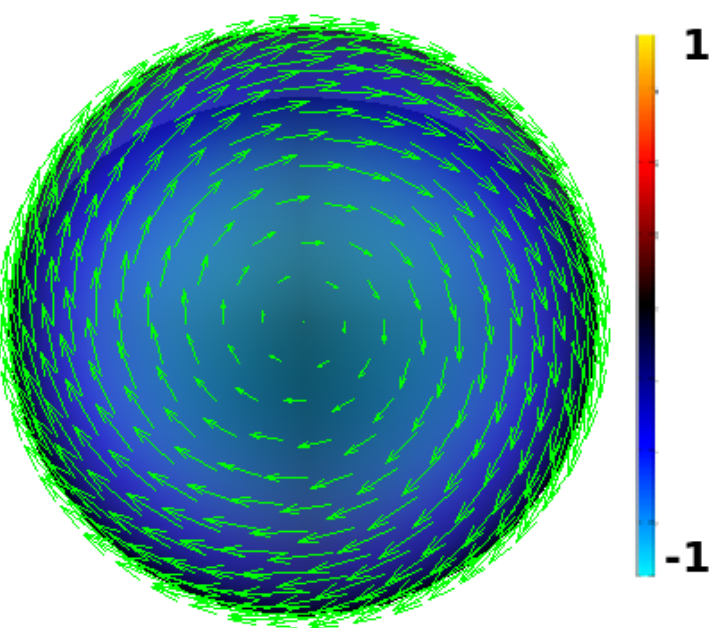

(b)

Figure 2: (a) Counter-clockwise rotating vector field, vortex identified by maxima in $A$. (b) Clockwise rotating vector field, vortex identified by minima in $A$. 
Secondly, HHD is tested on a classical bunny mesh as shown in Fig. 3. Vector fields containing sources and sinks was synthesized to mimic the optical flow of objects of increasing and decreasing sizes (shown in green arrows). Rotating vectors field was also generated to mimic the optical flow generated by a rotating vortex. The HHD of these vector fields was performed ( $U$ and $A$ are shown in overlapped textured color on the manifold).

Fig. 3(a) consists of a rotating vector field overlapped with A. Fig. 3(a) demonstrates that vortices of the original vector field can be identified using HHD on this arbitrary surface. Similarly, Fig. 3(b) contains diverging vector fields with $A$ being representing as textured color. Fig. 3(b) shows that sources and sinks of the vector field can also readily detected through the $U$ component of the HHD.

In Figs. 3(c) and 3(d), contains vector field of rotating and diverging components shown, and their corresponding $A$ and $U$ components respectively are represented as textured colors on the surface manifold. Figs. 3(c) and 3(d) demonstrate that different types of features in the same vector field can be identified by HHD. Our colormap shows sources and clockwise vortices in blue (local minima); sinks and counter-clockwise vortices in red (local maxima). 

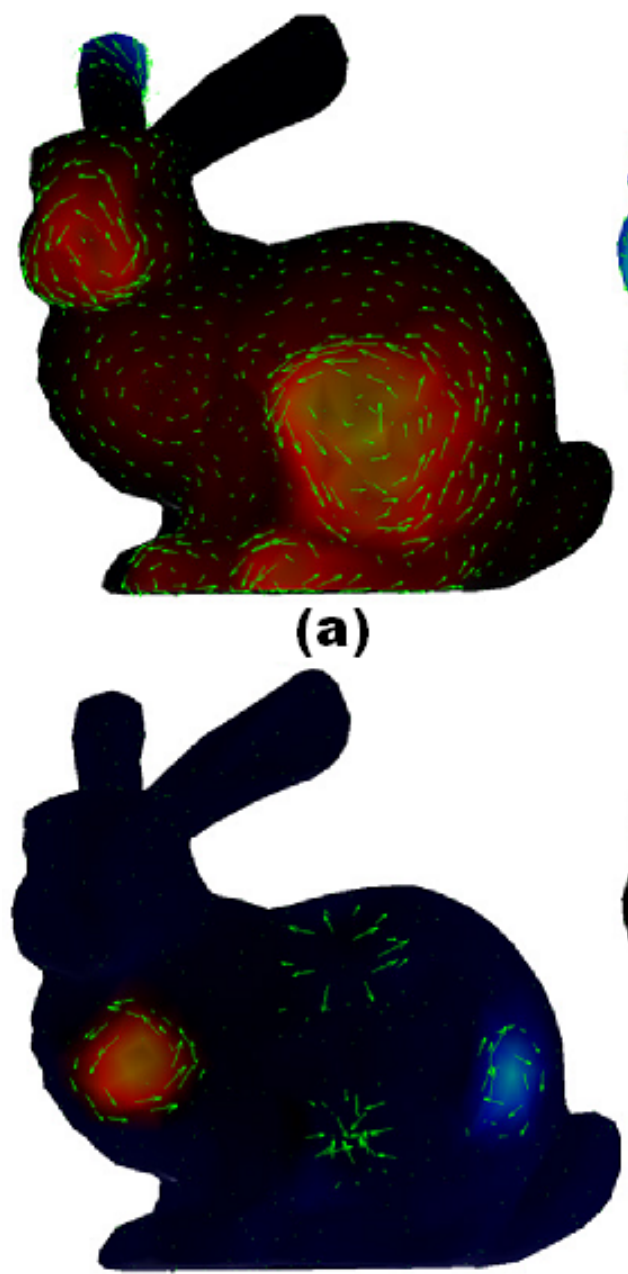

(c)

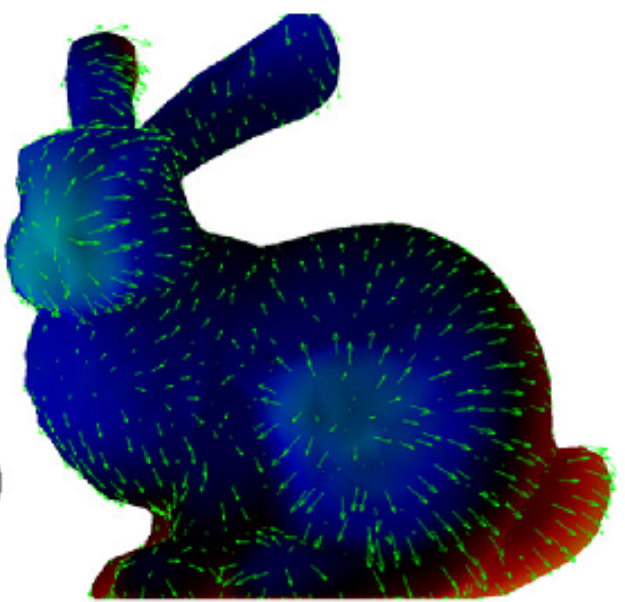

(b)

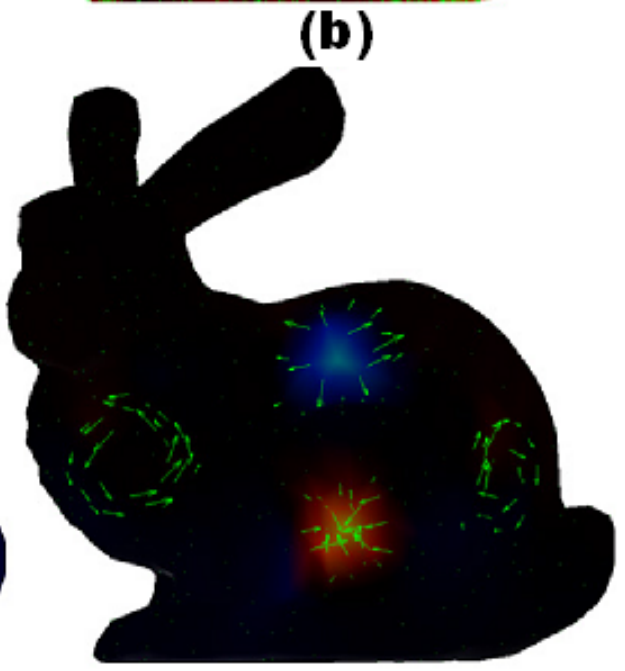

(d)

Figure 3: Vector fields are shown in green arrows and their $U$ and $A$ components are represented as textured color on the manifold: (a) Rotating vector field and its $A$ component; (b) Diverging vector field and its $U$ component; (c) Rotating and diverging vector field and its $A$ component; (d) Rotating and diverging vector field and its $U$ component.

In the last set of simulations, a source and a vortex were tracked on the surface of a bunny manifold, by finding critical points of the $U$ and $A$, HHD scalar fields (Fig. 4(a)). Time domain for the vortex can be considered as rotating vector field 
(like huricane) moving on a non flat manifold (surface of the earth). Furthermore, a surface patch of constant intensity was displaced using the advection equation, Lefèvre and Baillet (2008) and tracked through the vectors of the largest norm in vector field $\mathbf{H}$ (Fig. 4(b)). Possible real scenario for this simulation is tracking of an object (like automobile) on the non flat surface (like country side).

In Fig. 4 snapshots of tracking are overlaid on the surface and represented by symbols. A black arrow has been used to trace path of sources and vortex in Fig. 4(a) and moving the object in Fig. 4(b)

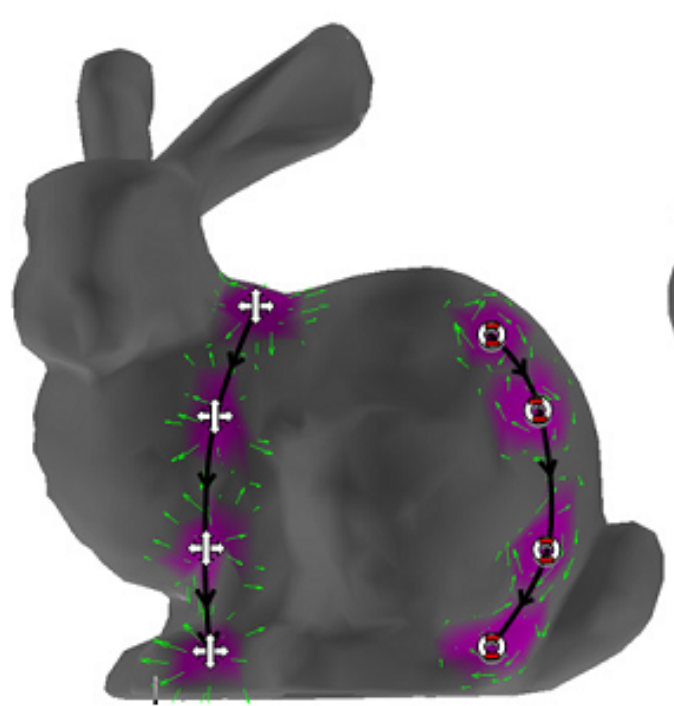

(a)

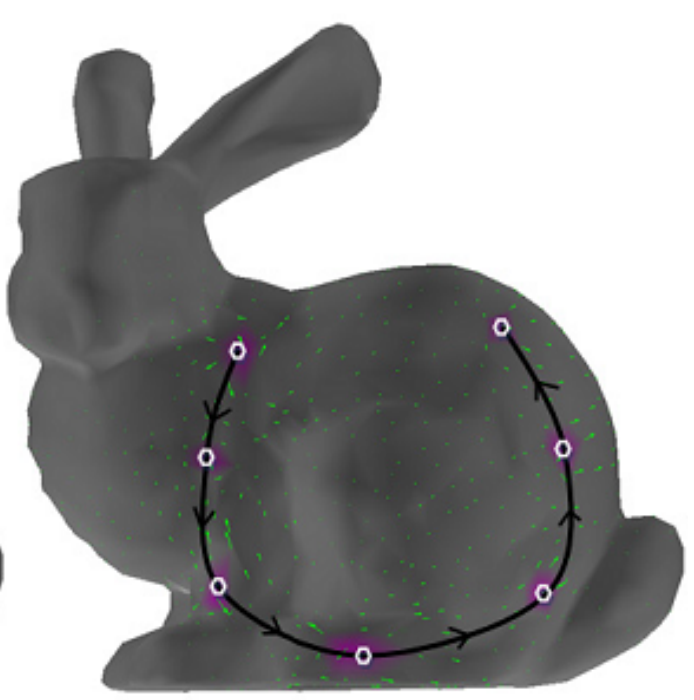

(b)

Figure 4: Tracking of sink, vortex and traveling surface patch of constant illumination on a rabbit surface manifold. Symbols have been assigned to each of these objects while arrows show the track they followed. Snapshots of tracking are overlaid to the figure: (a) Tracking of a source and a vortex; (b) Tracking of a patch of constant intensity. 
We further tested the HHD in a realistic scenario using experimental magnetoencephalography (MEG) data. The brain response was recorded over $306 \mathrm{MEG}$ sensors and the cortical currents were constrained on a surface tessellation of the subject's brain containing about 50,000 triangle nodes. The cortical currents were estimated with a regularized minimum-norm inverse model as detailed in Baillet et al. (2001). The vector field of the optical flow of these currents were computed using the method described in Lefèvre and Baillet (2008), as implemented by us in the BrainStorm (http://neuroimage.usc.edu/brainstorm). The image shows $U$ about $30 \mathrm{~ms}$ after stimulus onset, where the primary somatosensory brain response is expected, as shown with a strong divergent pattern over the central sulcus contralateral to the side of the stimulation.

Time resolved image series of cortical current sources can be obtained by reconstructing the generators of the magnetic fields that are measured outside the scalp (see Baillet et al. (2001) for an introduction). The optical flow from MEG source images representing motion fields of neural current on the surface of the brain was obtained from its optical flow. HHD was then applied to detect sources and sinks.

Results for the $U$ part of the HHD are shown in Fig. 5 to emphasis sources of cortical currents that evolves with time. A diverging source in the primary somatosensory cortex was found about $30 \mathrm{~ms}$ after the electrical stimulation of the contralateral index finger: this result was expected from the electrophysiology of the somatosensory systems. 


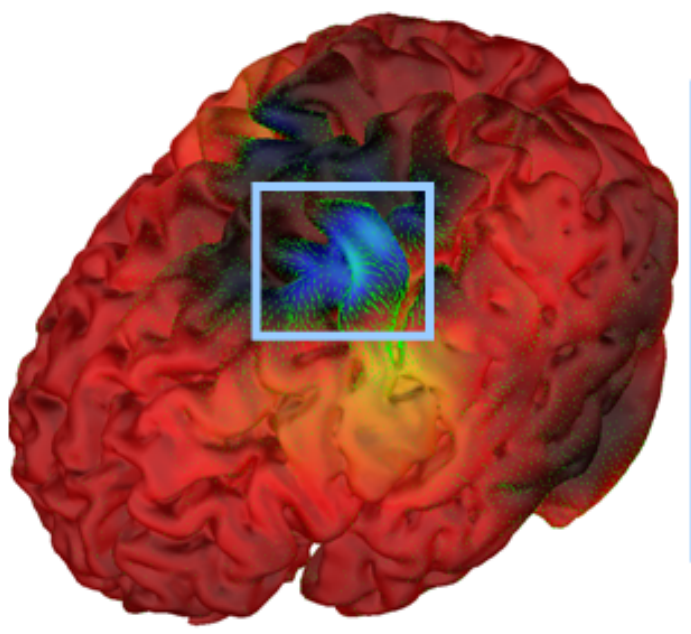

(a)

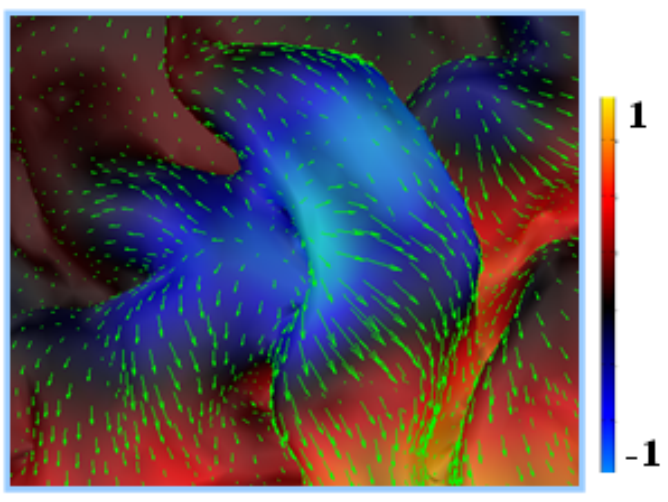

(b)

Figure 5: (a) Map of scalp potential $U$ obtained from the HHD of cortical currents estimated on the surface of the brain from MEG recordings following stimulation of the right median nerve. (b) Zoomed view of activation. Color map was scaled to arbitrary units of $U$ normalized to its instantaneous maximum.

\section{Conclusion and Future Work}

In this contribution, we developed a framework for the decomposition of a optical flow vector field on 2-Riemannian manifolds, which enabled us to successfully detect/track features on non-flat manifolds. The computations involved are simple, and been implemented in multi-threaded fashion which self distribute with respect to the computation cores available. The computation took less than 10 seconds to compute for all HHD components on a 50,000 node tessellation (Brain mesh, Fig. 5 ) on a conventional workstation having 8 cores. Evaluation of this framework under real and simulated environments revealed some promising applications in the emerging field of multi-dimensional imaging.

This contribution has also already started producing interesting results in med- 
ical imaging. Some applications of this tool in structural and functional neuroimaging are shown in Lefevre et al. (2009), Khan et al. (2009) and Khan et al. (2010). Applicability of this method on elephant manifolds are presented in supplementary material.

Future direction for this framework is its extension to the discretization using higher-order finite element analysis and utilization of this tool in more real world applications.

\section{Appendix}

First we will show that the scalar potentials $U$ and $A$ can be obtained up to a constant. If we consider two minimizers $U_{1}$ and $U_{2}$ for the functional in (5) then from equation (7) we get:

$$
\forall \phi \in H^{1}(\mathcal{M}), \int_{\mathscr{M}} g\left(\nabla_{\mathscr{M}}\left(U_{1}-U_{2}\right), \nabla_{\mathcal{M}} \phi\right) \mathrm{d} \mu=0
$$

Green's formula allows to transform this equation (given that $\mathcal{M}$ has no boundaries ) :

$$
\forall \phi \in H^{1}(\mathcal{M}), \int_{\mathscr{M}} \phi \operatorname{div}_{\mathcal{M}} \nabla_{\mathcal{M}}\left(U_{1}-U_{2}\right) \mathrm{d} \mu=0
$$

which corresponds to the Laplace equation:

$$
\Delta_{\mathscr{M}}\left(U_{1}-U_{2}\right)=0
$$

where $\Delta_{\mathcal{M}}=\operatorname{div}_{\mathcal{M}} \nabla_{\mathcal{M}}$ is the Laplace-Beltrami operator.

The same thing can be done for two minimizers $A_{1}$ and $A_{2}$ of the functional in 
(6). So, writing $R=U_{1}-U_{2}$ and $R^{\prime}=A_{1}-A_{2}$, we have the two conditions:

$$
\Delta_{\mathcal{M}} R=0, \Delta_{\mathcal{M}} R^{\prime}=0
$$

Then, multiplying by $R$ the first equation and integrating on the manifold we get:

$$
\int_{\mathcal{M}} R \Delta_{\mathscr{M}} R \mathrm{~d} \mu=0
$$

Green's formula again yields:

$$
\int_{\mathcal{M}} g\left(\nabla_{\mathcal{M}} R, \nabla_{\mathcal{M}} R\right) \mathrm{d} \mu=0
$$

Then it follows that $\nabla_{\mathscr{M}} R$ must equals zero and $R$ must be a constant. The same thing can be applied to $R^{\prime}$ which must be constant.

Secondly we will show the conditions on the vector field $\mathbf{H}$. Next if we write $\mathbf{H}=\mathbf{V}-\nabla_{\mathscr{M}} U+\mathbf{C u r l}_{\mathscr{M}} A$, we have:

$$
\operatorname{div}_{\mathcal{M}} \mathbf{H}=\operatorname{div}_{\mathcal{M}} \mathbf{V}-\operatorname{div}_{\mathcal{M}^{M}} \nabla_{\mathcal{M}} U \text { and } \operatorname{curl}_{\mathcal{M}} \mathbf{H}=\operatorname{curl}_{\mathcal{M}} \mathbf{V}-\operatorname{curl}_{\mathcal{M}} \operatorname{Curl}_{\mathcal{M}} A
$$

since $\operatorname{div}_{\mathcal{M}} \operatorname{Curl}_{\mathscr{M}} A=0$ and $\operatorname{curl}_{\mathcal{M}} \nabla_{\mathcal{M}} U=0$. Then:

$$
\forall \phi \in H^{1}(\mathcal{M}), \int_{\mathscr{M}} \phi \operatorname{div}_{\mathcal{M}} \mathbf{H d} \mu=\int_{\mathscr{M}} \phi\left(\operatorname{div}_{\mathcal{M}} \mathbf{V}-\operatorname{div}_{\mathcal{M}} \nabla_{\mathscr{M}} U\right) \mathrm{d} \mu
$$

And by using Green's formula and equation (7) we obtain:

$$
\forall \phi \in H^{1}(\mathcal{M}), \int_{\mathscr{M}} \phi \operatorname{div}_{\mathcal{M}} \mathbf{H} \mathrm{d} \mu=\int_{\mathscr{M}} g\left(\mathbf{V}, \nabla_{\mathcal{M}} \phi\right)-\int_{\mathscr{M}} g\left(\nabla_{\mathcal{M}} U, \nabla_{\mathcal{M}} \phi\right) \mathrm{d} \mu=0
$$


So we have proved that $\operatorname{div}_{\mathscr{M}} \mathbf{H}=0$ and the same strategy can be applied to obtain $\operatorname{curl}_{\mathcal{M}} \mathbf{H}=0$.

\section{References}

Baillet, S., Mosher, J., Leahy, R., 2001. Electromagnetic brain mapping. IEEE Signal Processing Magazine 18(6), 14-30.

Chorin, A., Marsden, J., 1993. A mathematical introduction to fluid mechanics. Springer.

Corpetti, T., Mémin, E., Pérez, P., 2003. Extraction of singular points from dense motion fields: an analytic approach. Journal of Mathematical Imaging and Vision 19, 175-198.

Do Carmo, M., 1993. Riemannian Geometry. Birkhäuser.

Druet, O., Hebey, E., Robert, F., 2004. Blow-up theory for elliptic PDEs in Riemannian geometry. Princeton University Press, Princeton, N.J., Ch. Background Material, pp. 1-12.

Guo, Q., Mandal, M., Li, M., 2005. Efficient Hodge-Helmholtz decomposition of motion fields. Pattern Recognition Letters 26 (4), 493-501.

Guo, Q., Mandal, M., Liu, G., Kavanagh, K., 2006. Cardiac video analysis using Hodge-Helmholtz field decomposition. Computers in Biology and Medicine 36 (1), 1-20.

Horn, B., Schunck, B., 1981. Determining Optical Flow. Artificial Intelligence 17 (1-3), 185-203. 
Khan, S., Lefèvre, J., Baillet, S., 2009. Feature Extraction from Time-Resolved Cortical Current Maps using the Helmholtz-Hodge Decomposition. In: 15th Human Brain Mapping International Conference, Sanfranciso.

Khan, S., Lefèvre, J., Raghavan, M., Baillet, S., 2010. Applications of 2Riemannian Helmholtz-Hodge Decomposition to MEG Source Dynamics. In: 17th Int. Conference on Biomagnetism, Dubrovnik.

Lefèvre, J., Baillet, S., June 2008. Optical flow and advection on 2-riemannian manifolds: a common framework. IEEE Transactions on Pattern Analysis \& Machine Intelligence 30 (6), 1081-92.

URL http://cogimage.dsi.cnrs.fr/publications/2008/LB08

Lefevre, J., F. Leroy, S. K., Dubois, J., Huppi, P., Baillet, S., Mangin, J., 2009. Identification of Growth Seeds in the Neonate Brain through Surfacic Helmholtz Decomposition. In: 21st Information Processing in Medical Imaging, Williamsburg.

Mann, S., Rockwood, A., 2002. Computing Singularities of 3D Vector Fields with Geometric Algebra. Proc. IEEE Visualization, 283-289.

Palit, B., Basu, A., Mandal, M., 2005. Applications of the Discrete Hodge Helmholtz Decomposition to Image and Video Processing. Lecture Notes in Computer Science 3776, 497.

Petronetto, F., Paiva, A., Lage, M., Tavares, G., Lopes, H., Lewiner, T., 2009. Meshless Helmholtz-Hodge Decomposition. IEEE Transactions on Visualization and Computer Graphics, 338-349. 
Polthier, K., Preuss, E., 2003. Identifying vector fields singularities using a discrete hodge decomposition. Visualization and Mathematics 3, 113-134.

Scheuermann, G., Tricoche, X., 2005. Topological Methods for Flow Visualization. The Visualization Handbook, 341-356.

Tong, Y., Lombeyda, S., Hirani, A., Desbrun, M., 2003. Discrete multiscale vector field decomposition. ACM Transactions on Graphics 22 (3), 445-452. 


\section{Supplementary Material for Pattern Recognition Letters}

Feature Detection and Tracking in Optical Flow on Non-Flat

\section{Manifolds}

In this supplementary material we will show application of a Modified HelmholtzHodge Decomposition (HHD) on elephant manifolds.

Fig. 1 shows result in the same spirit as Figs. 3(c) and 3(d). Vector fields are shown with green arrows. Fig. 1(a) contains vector field consist of rotating and diverging components. Fig. 1(c) shows same vector field but rotating component 3 and 4 are now identified using $A$ component of HHD overlapped on to the surface as texture color. In Fig. 1(d) sources 1 and sink 4 is identified by $U$ component of HHD. Fig. 1(c) and Fig. 1(c) demonstrate different types of features in same vector field can be identified by HHD. Our colormap shows sources and clockwise vortices in blue (local minima); sinks and counter-clockwise vortices in red (local maxima). Fig. 1(b) represent zoom image of sources and sink detected in Fig. 1(c) and Fig. 1(d). 


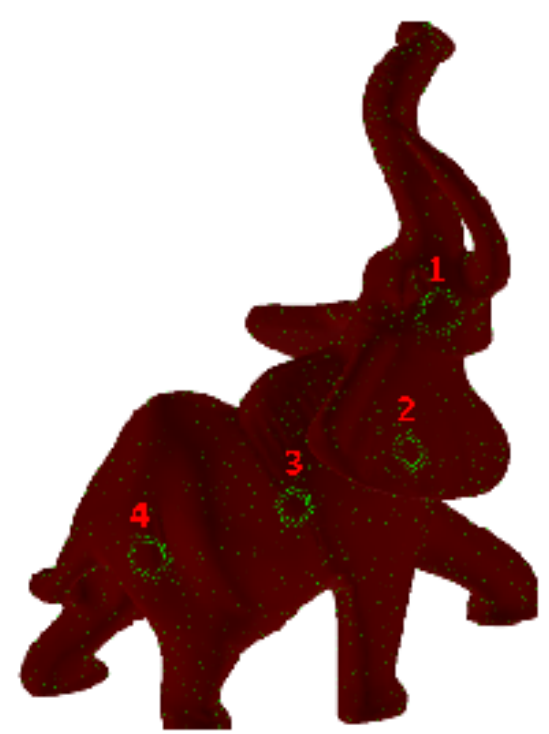

(a)

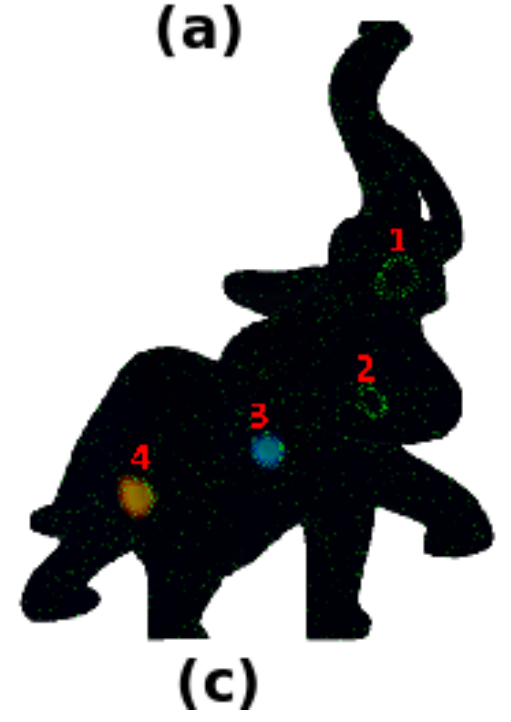

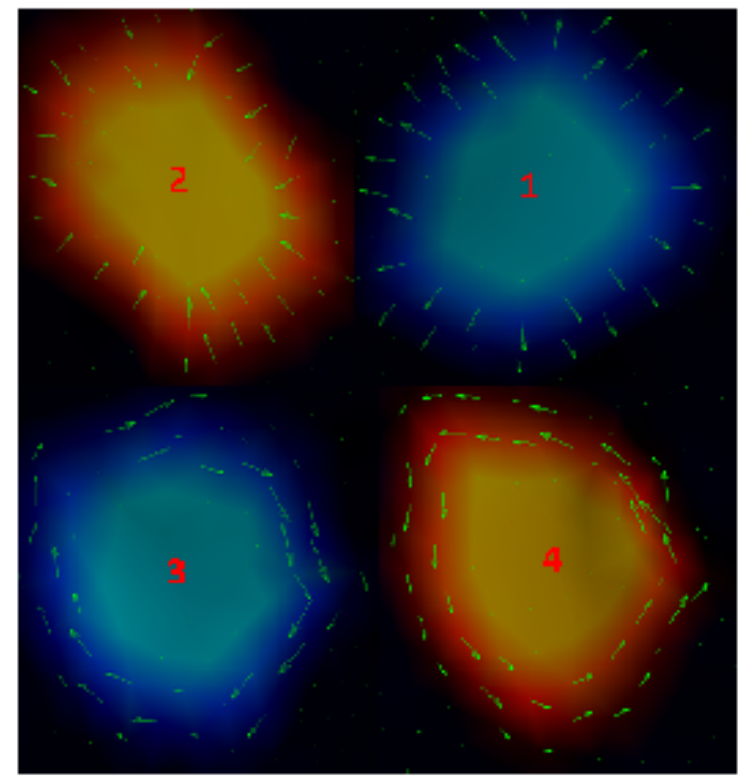

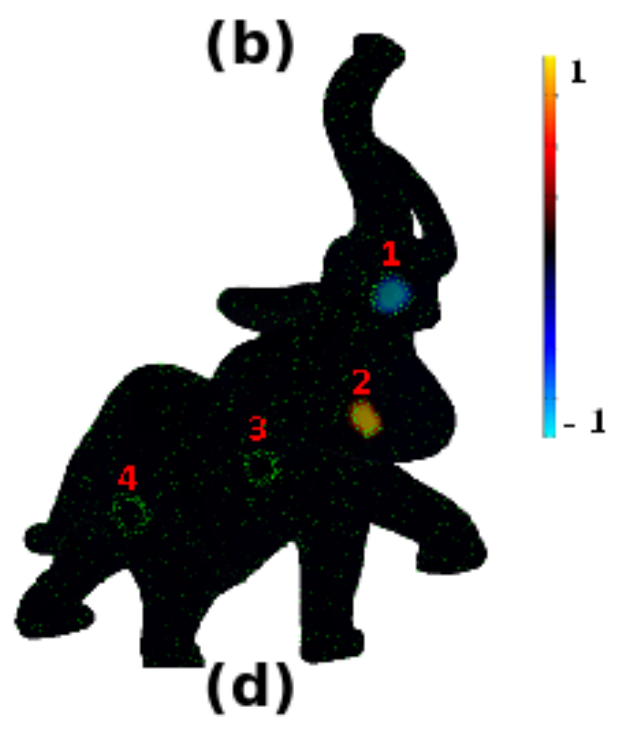

Figure 1: HHD on elephant manifold, vector field and its $U$ and $A$ components; Vortices, sources and sinks are identified by local maxima/minima of $U$ and $A$, extracted through HHD (a) Vector field on elephant surface; (b) Close-up view of vector field with $U$ and $A$ superimposed; (c) Rotating vector field detected by $A$ Component; (d) Diverging vector field identified by $U$. 